\title{
A Comparison of A Second-order Snow Model With Field Observations
}

\author{
Jinyang Du ${ }^{1}$, Jiancheng Shi ${ }^{1,2}$, Shengli $\mathrm{Wu}^{1}$ \\ 1.The Institute of Remote Sensing Applications, Chinese Academy of Sciences \\ P.O.Box9718,Beijing 100101, China \\ sunnydjy@hotmail.com \\ 2. Institute for Computational Earth System Science, University of California, Santa Barbara, U.S.A
}

\begin{abstract}
A microwave scattering model based on second-order solution of radiative transfer equation has been developed for dry snow. Advanced integral equation model (AIEM) and a semiempirical model were included in the model to account for ground contribution. Also, ellipsoid grain shape was adopted to describe ice particle. This model was compared with the groundbased scatterometer data (frequencies are $1.25 \mathrm{GHz}$ and $15.5 \mathrm{GHz}$ ) from NASA Cold-land Processes Field Experiment (CLPX). Inputs to the model were from Local-Scale Observation Site (LSOS) snow pit measurements, except that particle size and shape were computed as free parameters. The comparison shows that the model agrees well with the field data. Also from the comparison, it could be seen that particle shape had a significant effect on the cross-polarization signals.
\end{abstract}

Keywords- snow; second-order model; CLPX

\section{INTRODUCTION}

Active microwave remote sensing can provide useful information on snow parameters, such as snow cover extent and snow water equivalent (SWE) or the product of snow density and depth, for hydrological, climatological, and meteorological applications. In order to understand dry snow scattering behavior, theoretical models, many of which are based on radiative transfer theory, have been developed. For an optically thin layer of snow, single scattering model, which is based on first-order solution of radiative transfer equation, is applicable. However, as the optical depth of snow layer increases, multiple scattering models should be considered. Another factor affecting theoretical simulation results is the description of particle shape. Ice particle is generally modeled as spherical particles. However, the actual shape of ice particle is generally non-spherical and non-spherical particles can lead to strong depolarization return. In this paper, a second-order model, with ellipsoid scatterer considered, is described and compared with measured data.

\section{MODEL DESCRIPTION}

The proposed scattering model for dry snow was based on second-order solution of radiative transfer equation. And ellipsoid scatterer, which has the same volume as the sphere defined by input radius, was assumed when calculating phase matrix of snow. The advanced Integral Equation Model
(AIEM) [1] is used in the calculation of the backscattering of subsurface and air-snow interface as well as the interactions between ground and snow. It is noted that the surface backscattering signals for cross-polarization were estimated by an empirical model [2]. The received signal calculated by the model contains the following scattering mechanisms: (a) direct backscattering from air-snow interface, (b) direct backscattering from ground, (c) second-order volume scattering, (d) downward scattering by the particles followed by the coherent scattering by the ground, (e) coherent scattering by the ground followed by the scattering of the particles, (f) coherent scattering by the ground, followed by the scattering of the particles and another coherent scattering of the ground, (g)scattering from the particles followed by non-coherent scattering by the ground, (h) non-coherent scattering by the ground followed by scattering from the particles. The last five mechanisms could be regarded as interaction term $\mathrm{T}^{\mathrm{gv}}$ and the first three mechanisms belong to two surface terms $T^{\mathrm{a}}, \mathrm{T}^{\mathrm{g}}$ and one volume term $\mathrm{T}^{\mathrm{v}}$ respectively. The mathematically expressions of $\mathrm{T}^{\mathrm{a}}, \mathrm{T}^{\mathrm{g}}$ and $\mathrm{T}^{\mathrm{gv}}$ could be expressed as the following equations.

$$
\begin{aligned}
& T^{a}=S_{R 01}\left(\mu_{0 i}, \pi+\phi_{0 i} ;-\mu_{0 i}, \phi_{0 i}\right) \\
& T^{g}=T_{10}\left(\mu_{r}\right) \cdot S_{R 12}\left(\mu_{r}, \pi+\phi_{i} ;-\mu_{r}, \phi_{i}\right) \\
& \cdot T_{01}\left(\mu_{0 i}\right) \cdot \exp \left[-2 \kappa_{e} d / \mu_{r}\right] \\
& T^{g v}=\frac{1}{\mu_{i}} \mathbf{T}_{10}\left(\mu_{r}\right) \cdot \mathbf{T}_{01}\left(\mu_{i}\right) \cdot \\
& \left\{\operatorname { e x p } ( - \frac { 2 \kappa _ { e } d } { \mu _ { r } } ) \cdot \left[\mathbf{P}\left(-\mu_{r}, \pi+\phi_{i} ; \mu_{r}, \phi_{i}\right)\right.\right. \\
& \cdot \frac{1-\exp \left(-2 \kappa_{e} d / \mu_{r}\right)}{2 \kappa_{e}} \mathbf{R}_{12}^{2}\left(\mu_{r}\right)+ \\
& \mathbf{R}_{12}\left(\mu_{r}\right)\left[\mathbf{P}\left(-\mu_{r}, \pi+\phi_{i} ;-\mu_{r}, \phi_{i}\right)\right. \\
& \left.\left.+\left(\exp \left(\kappa_{2} d\right)-1\right) / \kappa_{2} \cdot \mathbf{P}\left(\mu_{r}, \pi+\phi_{i} ; \mu_{r}, \phi_{i}\right)\right]\right] \\
& +\int_{0}^{1} \frac{\exp \left(-\kappa_{1} d\right)}{\mu^{\prime}} d \mu^{\prime} \int_{0}^{2 \pi} d \phi^{\prime} \frac{1-\exp \left(-\kappa_{2} d\right)}{\kappa_{2}} \\
& \cdot \mathbf{P}\left(\mu_{i}, \pi+\phi_{i} ; \mu^{\prime}, \phi^{\prime}\right) \cdot \mathbf{S}_{R 12}\left(\mu^{\prime}, \phi^{\prime} ; \mu_{r}, \phi_{i}\right)
\end{aligned}
$$




$$
\begin{aligned}
& +\frac{\exp \left(\kappa_{2} d\right)-1}{\kappa_{2}} \mathbf{S}_{R 12}\left(\mu_{r}, \pi+\phi_{i} ; \mu^{\prime}, \phi^{\prime}\right) \\
& \left.\cdot \mathbf{P}\left(-\mu^{\prime}, \phi^{\prime} ;-\mu_{r}, \phi_{i}\right)\right\}
\end{aligned}
$$

where $\mathrm{S}$ is the phase matrix of surface calculated by AIEM, T is the transmitivity matrix, $\mathrm{R}$ is the reflectivity matrix, $\mathrm{P}$ is the phase matrix of snow, the subscripts $0,1,2$ in $S, T$ and $R$ denote air, snow and ground medium respectively, $d$ is the depth of snow layer and $\kappa_{1}=\kappa_{e}\left(1 / \mu_{i}+1 / \mu^{\prime}\right), \kappa_{2}=\kappa_{e}\left(1 / \mu_{i}-1 / \mu^{\prime}\right)$. Volume term $\mathrm{T}^{\mathrm{v}}$ could be derived from two iterative processes when solving the radiative transfer equation [3], [4]. It is noted that Rayleigh Approximation was applied to ellipsoid particles when calculating the phase matrix of snow [4].

To calculate scattering effects from surface, surface scattering model AIEM is applied. AIEM is the a recent development of Integral Equation Method (IEM) [5], which was verified by laboratory measurements of bistatic scattering from surfaces with small, intermediate and large scale roughness. AIEM kept the absolute phase term in Greens function, meaning better accuracy than the old IEM. According to AIEM, the bistatic scattering coefficient can be expressed as the sum of Kirchhoff term, complementary term and cross term, as (4) shows.

$$
\begin{aligned}
& \sigma_{q p}^{s}=\sigma_{q p}^{k}+\sigma_{q p}^{k c}+\sigma_{q p}^{c}=\frac{k^{2}}{2} \exp \left[-s^{2}\left(k_{z}^{2}+k_{s z}^{2}\right)\right] \\
& \sum_{n=1}^{\infty} s^{2 n}\left|I_{p q}^{n}\right|^{2} \frac{W^{(n)}\left(k_{s x}-k_{x}, k_{s y}-k_{y}\right)}{n !}
\end{aligned}
$$

where $\sigma_{q p}^{k}$ denotes Kirchhoff term, $\sigma_{q p}^{c}$ for complementary tem, $\sigma_{q p}^{k c}$ for cross term, pq denotes polarization state, $\mathrm{s}$ is the standard deviation of the surface height, $\mathrm{k}$ is wave number, $k_{x}=k \sin \theta \cos \phi, k_{y}=k \sin \theta \sin \phi, k_{s x}=k \sin \theta_{s} \cos \phi_{s}$, $k_{s y}=k \sin \theta_{s} \sin \phi_{s}, k_{z}=k \cos \theta, k_{s z}=k \cos \theta_{s}, \theta, \varphi$ are for incident direction, $\theta_{s}, \phi_{s}$ are for the scattered direction, $W^{n}$ is the Fourier transform of the nth power of a known surface correlation function. And detailed expression of $I_{p q}^{n}$ could be referred to [1]. Bistatic scattering coefficient $\sigma^{0}$ calculated by AIEM could be linked with surface phase matrix as (5) shows.

$$
I^{s}\left(\theta_{s}, \phi_{s}\right)=\frac{1}{4 \pi} \int\left[\frac{\sigma^{0}\left(\theta_{s}, \theta ; \phi_{s} \phi\right)}{\cos \theta_{s}}\right] I^{i}(\theta, \phi) d \Omega
$$

where $I^{s}$ and $I^{i}$ are scattering and incident intensity respectively.

\section{FIELD MEASUREMENTS}

In order to verify the proposed model, data from NASA Cold-land Processes Field Experiment (CLPX) were used for comparison. The data were measured by University of Michigan truck-mounted $\mathrm{L}$ band $(1.25 \mathrm{GHz})$ and $\mathrm{Ku}$ band $(15.5 \mathrm{GHz})$ scatterometers during the third Intensive Observation Period (IOP3, dry snow), February 19 -25, 2003 at the Local Scale Observation Site (LSOS) test site. The test site consisted of a small ( 0.8 -ha) clearing surrounded by trees and located within the CLPX Fraser Intensive Study Area (ISA), near the Fraser Experimental Forest Headquarters Facility, Colorado. The incidence angles are 20, 35 and 50 degrees. Snow input parameters were from snow pits measurements, which were taken at the same time as the ground-based scatterometer measurements, at LSOS except that particle size and shape were computed as free parameters. Actually, measurements on particle size along the snow-pack vertical profile were available, but the proposed one-layer model needs an effective radius, which should be the radius having particle size distribution and near-field effects considered. Similarly, the shape used in simulation could be regarded as an effective shape. The contribution from the airsnow surface is assumed to be negligible since the discontinuity between air and dry snow is normally small. Also, since there is a lack of ground information and snow contribution is quite small at L-Band in this case, comparisons between simulated data with variant ground situations and measured data were made in order to estimate ground parameters. The ground and snow inputs could thus be concluded as the following:

- Ground rms-height $0.5 \mathrm{~cm}$

- Ground surface correlation length $10 \mathrm{~cm}$

- Soil moisture $25 \%$

- Snow depth 0.99 meter

- Snow volume fraction $22.4 \%$.

\section{SIMULATION RESULTS AND DISCUSSION}

As stated above, comparison with the measurement at Lband is used to find proper ground parameters. Fig. 1 shows the result of comparison between the model and the measurement on the morning of Feb 23, 2003 at L-Band. Grain radius is $0.46 \mathrm{~mm}$ and a prolate ellipsoid with 0.7 as the ratio of short axis to long axis is considered. Fig. 2 shows the comparison result at $\mathrm{Ku}$ band. Particle size and shape were best-fit parameters in this case. A $0.42 \mathrm{~mm}$ radius and 0.1 short axis to long axis ratio were selected in the simulation.

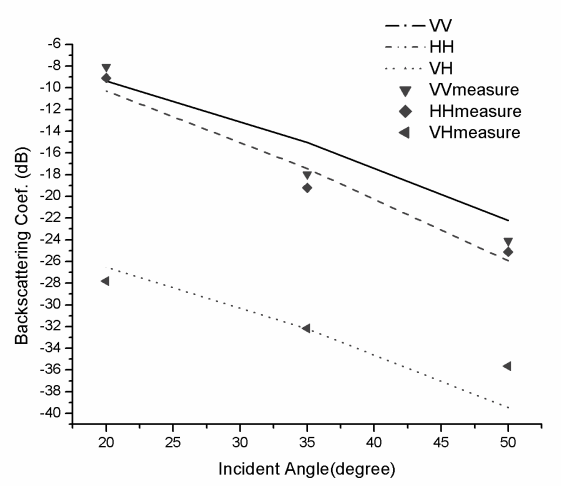

Figure 1. Comparison between proposed model and measured data at LBand (scatter points are for measured data) 


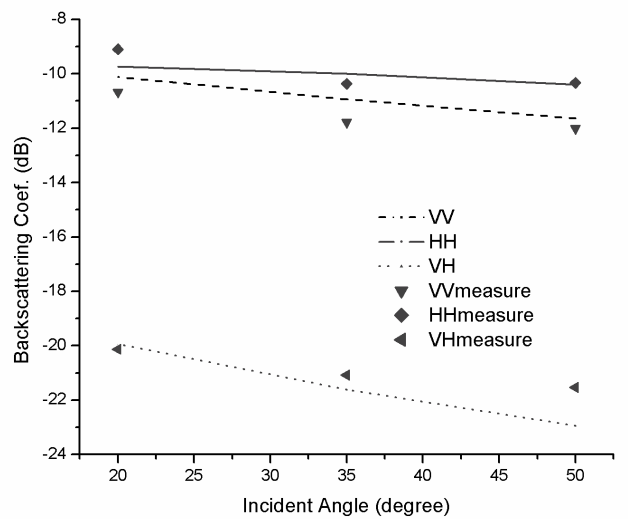

Figure 2. Comparison between proposed model and measured data at $\mathrm{Ku}-$ Band (scatter points are for measured data. short axis to long axis ratio is 0.1 )

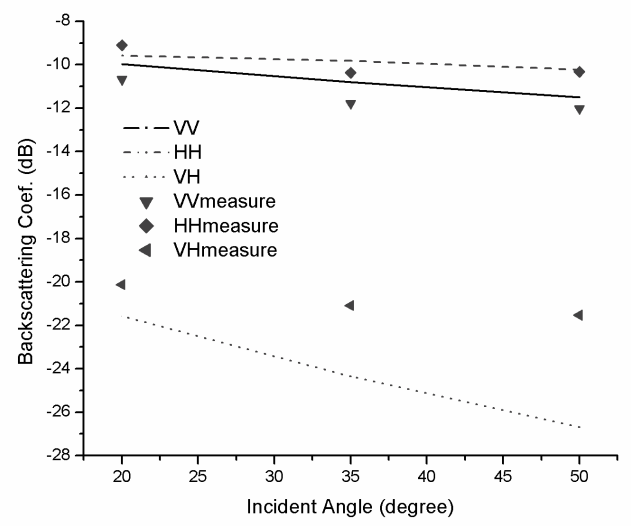

Figure 3. Comparison between proposed model and measured data at $\mathrm{Ku}-$ Band (scatter points are for measured data. short axis to long axis ratio is 0.7 )

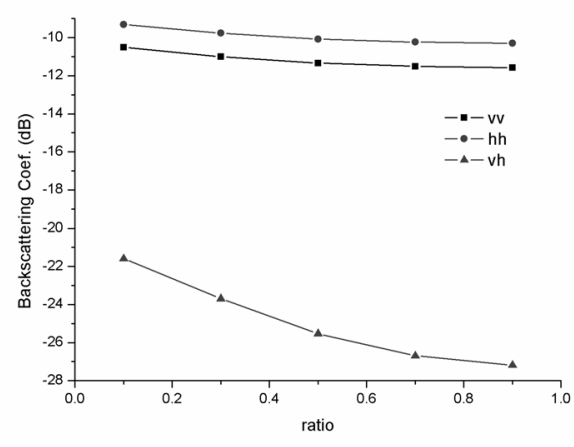

Figure 4. Backscattering coefficients trend as particle shape changes (x-axis is the short axis to long axis ratio of ellipsoid)
It can be seen from Fig. 1 that simulation agrees well with the measured data at L-band with the selected ground parameters. The selected ground parameters were then used in Ku-band simulations. Fig.2 shows that by selecting proper particle size and shape, which were the best-fit parameters to minimize the Mean Square Error (MSE) between simulated and measured backscattering coefficients, the simulation agrees quite well with measured data in terms of magnitudes for co-polarizations and cross-polarizations as well as the difference between VV and $\mathrm{HH}$ polarizations.

In order to illustrate the effects of particle shape on snow backscattering, simulation was made by considering a slightly larger but more sphere-like snow particle as Fig. 3 shows. Fig. 3 only differs from Fig. 2 in that a 0.7 short axis to long axis ratio and $0.46 \mathrm{~mm}$ radius were adopted. By comparing the two figures, it can be seen that particle shape have a significant effect on snow backscattering, especially for crosspolarization. The needle-like particles can produce much more cross-polarization return than sphere-like particles and this is more evident as Fig. 4 shows, which shows how backscattering coefficients change with ellipsoid shape.

\section{CONCLUSION}

A second-order model for snow was described and comparisons were made between the model and measured data from CLPX. Model inputs were represented by the average values of measured snow parameters, with the exception of the particle radius and shape, which were regarded as effective values to the model. The model shows good agreement with the measured data. Also from the comparison, it can be seen that particle shape has a significant effect on snow backscattering signals, especially for the cross-polarization.

\section{REFERENCES}

[1] K. S. Chen, T. D. Wu, et al., "Emission of rough surfaces calculated by the Integral Equation Method with comparion to three-dimensional moment method simulations", IEEE trans. Geosci. Remote Sens., vol. 41, no. 3, pp. 90-101, 2003.

[2] Y. Oh, K. Sarabandi, and F.T. Ulaby, "An empirical model and an inversion technique for radar scattering from bare soil surfaces," IEEE Trans. Geosci. Remote Sensing, vol. 30, pp. 370381, March 1992.

[3] J. Shi and J. Dozier, "Estimation of Snow Water Equivalence Using SIR-C/X-SAR, Part II: Inferring snow depth and particle size", IEEE Transactions on Geoscience and Remote Sensing, Vol 38, No. 6, pp. 2475-2488, Nov. 2000.

[4] L. Tsang, J.A. Kong and R.T. Shin, Theory of Microwave Remote Sensing. New York: Wiley-Interscience, 1985.

[5] A. K. Fung, Microwave Scattering and Emission Models and Their Applications. Norwood, MA: Artech House, 1994 